\title{
Kein perfektes Wahlsystem, aber ein guter Kompromiss - unter schwierigen Rahmenbedingungen ${ }^{1}$
}

\section{Einleitung}

Die »Lebensdauer « des Bundeswahlgesetzes in der Fassung des Neunzehnten Gesetzes zur Änderung des Bundeswahlgesetzes war kurz, sehr kurz - sogar so kurz, dass es nur "auf dem Papier« existierte und nie bei einer Bundestagswahl zur Anwendung kam. Rückblende: Am 3. Juli 2008 hat das Bundesverfassungsgericht - durchaus überraschend - Teile des Bundeswahlgesetzes für verfassungswidrig erklärt und bis spätestens 30. Juni 2011 die Behebung des verfassungswidrigen Zustands verlangt. ${ }^{2}$ Nachdem die Große Koalition in der 16. Wahlperiode des Deutschen Bundestages (2005-2009) nicht mehr tätig geworden war und in der darauffolgenden Wahlperiode die gesetzte Frist verstrichen und ein Kompromiss zwischen Regierungs- und Oppositionsparteien nicht möglich war, ${ }^{3}$ hat die CDU/CSU-FDP-Regierungskoalition am 29. September 2011 »im Alleingang « über ein neues Bundeswahlgesetz entschieden. Mit der »schwarz-gelben Wahlrechtsreform « wurde die Möglichkeit der Listenverbindung abgeschafft und eine Sitzverteilung nach Ländersitzkontingenten eingeführt, wobei die Bestimmung der Größe dieser Sitzkontingente auf der Grundlage der Wählerzahl vorgesehen war. Zudem wurde eine Reststimmenverwertung bzw. Vergabe von Zusatzmandaten für nicht verwertete Reststimmen in den einzelnen Ländern eingeführt, wobei ausschließlich die Verrechnung der jeweiligen Abrundungsverluste vorgesehen war. ${ }^{4}$ Gegen das am 3. Dezember 2011 in Kraft getretene novellierte Bundeswahlgesetz wurde erneut vor dem Bundesverfassungsgericht geklagt, was angesichts der vorangegangenen Debatte und der starken Mo-

1 Der Beitrag basiert im Wesentlichen auf meiner Stellungnahme, die ich als Sachverständiger für die öffentliche Anhörung des Innenausschusses des Deutschen Bundestages am 14. Januar 2013 abgegeben habe (A-Drs. 17(4)634 H).

2 Vgl. BVerfG, 2 BvC 1/07, 2 BvC 7/07 vom 3.7.2008.

3 Es war nicht nur kein Kompromiss zwischen Regierungs- und Oppositionsparteien möglich. Auch innerhalb der Opposition konnte kein Kompromiss hergestellt werden, zumal jede Oppositionspartei einen eigenen Gesetzentwurf einbrachte. Vgl. dazu ausführlich: Gerd Strohmeier, »Die schlechteste Wahlsystemreform - mit Ausnahme aller anderen« in: Zeitschrift für Politik, 58 (2011) 4, S. 393-409.

4 Vgl. BGBl. 2011, Teil 1 Nr.60, S. 2313; Gerd Strohmeier, Die schlechteste Wahlsystemreform mit Ausnahme aller anderen, aaO. (FN 3), S. 397-402. 
bilisierung gegen das reformierte Sitzzuteilungsverfahren (im weiteren Sinne) $)^{5}$ nicht wirklich überraschen konnte. Für eine große Überraschung sorgte indessen (wieder einmal) das Bundesverfassungsgericht, das am 25. Juli 2012 erneut Teile des (novellierten) Bundeswahlgesetzes für verfassungswidrig erklärte, dabei nicht unerheblich von früheren Entscheidungen abwich sowie eine neuerliche Reform des Bundeswahlgesetzes notwendig machte ${ }^{6}$ - und hierfür den verfassungsrechtlichen Spielraum zur Gestaltung des Sitzzuteilungsverfahrens weiter einschränkte. Angesichts der Tatsache, dass der Wunsch bzw. die Forderung nach einem »All-Parteien-Kompromiss « bzw. nach einer breiten parlamentarischen Mehrheit, bestehend aus Regierungs- und Oppositionsparteien, für die erneut notwendige Reform des Bundeswahlgesetzes relativ hoch und die Kompromissmöglichkeiten für diese Reform verhältnismäßig klein waren, war die Ausgangssituation nach dem Bundesverfassungsgerichtsurteil vom 25. Juli 2012 alles andere als einfach. Schließlich war es aufgrund der vielfältigen verfassungsrechtlich notwendigen und verfassungspolitisch gewollten Ziele, zwischen denen zum Teil erhebliche Zielkonflikte bestehen, äußerst schwierig, einen für alle (prinzipiell kompromissbereiten) Fraktionen akzeptablen Kompromiss zu finden.

Im Folgenden wird zunächst das Urteil des Bundesverfassungsgerichts vom 25. Juli 2012 bzw. der daraus folgende Regelungsauftrag beschrieben. Anschließend wird das von den Fraktionen der CDU/CSU, SPD, FDP und Bündnis 90/Die Grünen eingebrachte und beschlossene Zweiundzwanzigste Gesetz zur Änderung des Bundeswahlgesetzes - kurz: das Modell "Sitzkontingente plus Ausgleich « - erläutert und vor dem Hintergrund der verschiedenen verfassungsrechtlich notwendigen und verfassungspolitisch gewollten Ziele bewertet. Anschließend erfolgt eine (vergleichende) Erläuterung und Bewertung des von der Fraktion Die Linke eingebrachten Modells »Kompensation plus Ausgleich «?

\section{Das Urteil des Bundesverfassungsgerichts vom 25. Juli 2012}

Das Bundesverfassungsgericht hat in seinem Urteil vom 25. Juli 2012 entschieden, dass » $\$ 6$ Absatz 1 Satz 1 [die Bestimmung der Sitzkontingente der Länder] und Absatz 2a des Bundeswahlgesetzes [die Reststimmenverwertung bzw. Vergabe von Zusatzmandaten] in der Fassung des Neunzehnten Gesetzes zur Änderung des Bundeswahlgesetzes vom

5 Mit dem Sitzzuteilungsverfahren im weiteren Sinne wird der gesamte Prozess der Verrechnung von Stimmen in Mandate bezeichnet, nicht nur das Stimmenverrechnungsverfahren (z.B. das Sainte-Laguë/Schepers-Verfahren), das sich als Sitzzuteilungsverfahren im engeren Sinne bezeichnen lässt.

6 Vgl. BVerfG, 2 BvF 3/11, 2 BvR 2670/11, 2 BvE 9/11 vom 25.7.2012.

7 Bei der Erläuterung und Bewertung der beiden Modelle erfolgt eine Konzentration auf die wesentlichen Gesichtspunkte. Weitere Sitzzuteilungsverfahren, die im Vorfeld auf den Sitzungen der interfraktionellen Arbeitsgruppe zur Wahlrechtsreform diskutiert wurden, z.B. das Modell »Sitzkontingente plus 50« oder das Modell »Pukelsheim III« (vgl. Richard Peifer / Daniel Lübbert / Kai-Friederike Oelbermann / Friedrich Pukelsheim, »Direktmandatsorientierte Proporzanpassung: Eine mit der Personenwahl verbundene Verhältniswahl ohne negative Stimmgewichte« in: DVBl, 127 (2012) 12, S. 725-792), werden an dieser Stelle nicht behandelt. 
25. November 2011 [...] mit Artikel 21 Absatz 1 und Artikel 38 Absatz 1 Satz 1 des Grundgesetzes unvereinbar und nichtig $\ll^{8}$ sind sowie „\$6 Absatz 5 des Bundeswahlgesetzes [die Möglichkeit der Entstehung von Überhangmandaten] in der Fassung des Neunzehnten Gesetzes zur Änderung des Bundeswahlgesetzes vom 25. November 2011 [...] nach Maßgabe der Gründe mit Artikel 21 Absatz 1 und Artikel 38 Absatz 1 Satz 1 des Grundgesetzes unvereinbar « ${ }^{9}$ ist.

\subsection{Die Bestimmung der Sitzkontingente der Länder}

Die Bestimmung der Sitzkontingente der Länder nach der Wählerzahl ( $\$ 6$ Abs. 1 Satz 1 BWG) verletze »die Grundsätze der Gleichheit und Unmittelbarkeit der Wahl sowie der Chancengleichheit der Parteien ${ }^{10}{ }^{10}$ a sie den Effekt des negativen Stimmgewichts (besser, aber weniger gebräuchlich: inversen Erfolgswerts) »mit beachtlicher Wahrscheinlichkeit« - in » mindestens in etwa der gleichen Größenordnung $[\ldots]$ wie $[\ldots]$ im bisherigen Wahlrecht «- ermögliche. ${ }^{11}$ Dies kann (zumindest auf der Grundlage des Urteils des Bundesverfassungsgerichts vom 3. Juli 2008) aus guten Gründen anders gesehen werden. ${ }^{12}$ Entscheidend ist allerdings, dass das Bundesverfassungsgericht die Vergabe der Sitze nach Ländersitzkontingenten nicht grundsätzlich beanstandet hat, sondern ausschließlich die Bestimmung der Ländersitzkontingente auf der Grundlage der Wählerzahl, und dass nach der Rechtsprechung des Bundesverfassungsgerichts diese Bestimmungsgröße durch die Zahl der Bevölkerung oder der Wahlberechtigten substituiert werden kann: »Von Verfassungs wegen ist der Gesetzgeber nicht daran gehindert, diesen Ursachenzusammenhang [den Effekt des negativen Stimmgewichts] innerhalb des von ihm geschaffenen Wahlsystems zu unterbinden, indem er zur Bemessung der Ländersitzkontingente statt der Wählerzahl die Zahl der Bevölkerung oder der Wahlberechtigten heranzieht. Denn jede vom Wahlverhalten der Wahlberechtigten nicht beeinflusste Größe als Grundlage der Bestimmung der Ländersitzkontingente würde den Effekt des negativen Stimmgewichts bei der Sitzzuteilung vermeiden. «13

In diesem Zusammenhang ist darauf hinzuweisen, dass das Bundesverfassungsgericht in seinem Urteil vom 25. Juli 2012 - wie bereits in seinem Urteil vom 3. Juli $2008^{14}$ nicht nur den klassischen Tatbestand des negativen Stimmgewichts (»dass ein Zuwachs an Stimmen zu Mandatsverlusten führt« bzw. »dass für den Wahlvorschlag einer Partei

8 BVerfG, 2 BvF 3/11, 2 BvR 2670/11, 2 BvE 9/11 vom 25.7.2012, Tenor Nr. II.1.

9 Ebd., Tenor Nr. II.2.

10 Ebd., Rn. 66.

11 Ebd., Rn. 95.

12 Vgl. Strohmeier, Die schlechteste Wahlsystemreform - mit Ausnahme aller anderen, aaO. (FN 3), S. 397-399; Volker M. Haug, »Das Bundesverfassungsgericht als Gesetzgeber anstelle des Gesetzgebers: Ein kritischer Blick auf das Wahlrechtsurteil vom 25. Juli 2012 «in: ZParl, (2012) 3, S. 658-674, hier: S. 662-665; Christian Hesse, »Wahlrecht 2013 mit Schattenseiten. Oder: Ein kritisches Lob dem vom Bundesverfassungsgericht verworfenen Bundeswahlgesetz von 2011 «, in: ZParl, (2009) 1, S. 177-200, hier: S. 180-183, 187-194.

13 BVerfG, 2 BvF 3/11, 2 BvR 2670/11, 2 BvE 9/11 vom 25.7.2012, Rn. 96.

14 BVerfG, 2 BvC 1/07, 2 BvC 7/07 vom 3.7.2008, Rn. 103. 
insgesamt mehr Mandate erzielt werden, wenn auf ihn selbst weniger [...] Stimmen entfallen«), sondern auch einen erweiterten Tatbestand des negativen Stimmgewichts (»dass für den Wahlvorschlag einer Partei insgesamt mehr Mandate erzielt werden, wenn [...] auf einen konkurrierenden Vorschlag mehr Stimmen entfallen «) aufgeführt hat. ${ }^{15}$ Damit erfasst der vom Bundesverfassungsgericht beanstandete Effekt des negativen Stimmgewichts insgesamt den Tatbestand, »dass die Sitzzahl einer Partei erwartungswidrig mit der auf diese oder [sic] eine konkurrierende Partei entfallenden Stimmenzahl korreliert «. ${ }^{16}$ Schließlich hat das Gericht gefordert, dass ein Mandatszuteilungsverfahren »grundsätzlich frei von willkürlichen oder widersinnigen Effekten sein « müsse - und der Wähler vor dem Wahlakt erkennen können müsse, » wie sich die eigene Stimmabgabe auf Erfolg oder Misserfolg der Wahlbewerber auswirken kann ${ }^{17}{ }^{17}$

\subsection{Die Reststimmenverwertung bzw. Vergabe von Zusatzmandaten}

Die Reststimmenverwertung bzw. Vergabe von Zusatzmandaten ( $\$ 6$ Absatz 2a BWG) verletze »die Grundsätze der Wahlrechtsgleichheit und der Chancengleichheit der Parteien ${ }^{18}$ da sie »eine Ungleichbehandlung der Wählerstimmen « bewirke und »in die Chancengleichheit der Parteien« eingreife, »ohne dass dies durch einen besonderen, sachlich legitimierten Grund gerechtfertigt wäre «. ${ }^{19}$ Allerdings hat das Bundesverfassungsgericht die Reststimmenverwertung bzw. Vergabe von Zusatzmandaten nicht grundsätzlich beanstandet, sondern nur die konkrete Ausgestaltung durch $\$ 6$ Absatz $2 \mathrm{a}$ BWG: »Die Entscheidung, den durch die Unterteilung des Wahlgebietes in Listenwahlkreise herbeigeführten Proportionalitätseinbußen durch einen wahlgebietsbezogenen Verhältnisausgleich entgegenzuwirken, ist zwar von Verfassungs wegen nicht zu beanstanden [...]. Die Vergabe von Zusatzmandaten nach $\$ 6$ Abs. 2a BWG ist jedoch zur Erreichung dieses Zieles nicht geeignet. $\aleph^{20}$ Schließlich erfordere ein derartiger wahlgebietsbezogener Verhältnisausgleich, dass in den Listenwahlkreisen nicht nur die Abrundungsverluste, sondern »sowohl die Abrundungsverluste als auch die Aufrundungsgewinne der Landeslisten einer Partei identifiziert, wahlgebietsbezogen aufsummiert und anschließend miteinander verrechnet werden ${ }^{21}{ }^{21}$ Die Kritik des Bundesverfassungsgerichts ist an dieser Stelle nachvollziehbar und im Vorfeld der »schwarz-gelben Wahlrechtsreform « auch von deren Befürwortern vorgebracht worden. ${ }^{22} \mathrm{Da} \$ 6 \mathrm{Abs} .2 \mathrm{a}$ BWG nur die Abrundungsverluste berücksichtigt, ist er laut Bundesverfassungsgericht auch »nicht geeignet, eine mit den Überhangmandaten verbundene Verzerrung der Erfolgs-

15 BVerfG, 2 BvF 3/11, 2 BvR 2670/11, 2 BvE 9/11 vom 25.7.2012, Rn. 85.

16 Ebd.

17 Ebd., Rn. 57.

18 Ebd., Rn. 98.

19 Ebd., Rn. 101.

20 Ebd., Rn. 104.

21 Ebd., Rn. 105.

22 Vgl. Strohmeier, Die schlechteste Wahlsystemreform - mit Ausnahme aller anderen, aaO. (FN 3), S. 400.

ZfP 60. Jg. 2/2013 
wertgleichheit auszugleichen «. ${ }^{23}$ Zudem sei er nicht durch das Ziel zu rechtfertigen, »die durch die Unterteilung des Wahlgebietes [...] in kleinen Ländern herbeigeführte faktische Sperrwirkung [...] zu kompensieren «, da die Zusatzmandate »nicht zielgenau an Parteien vergeben « werden, »deren Landeslisten von einer das gesetzliche Quorum überschreitenden effektiven Sperrwirkung betroffen sind, sondern [...] an prinzipiell jede Partei ${ }^{24}$

\subsection{Die Möglichkeit der Entstehung von Überhangmandaten}

Grundsätzlich hat das Bundesverfassungsgericht - wie bereits in früheren Urteilen festgestellt, dass "[d]ie mit der ausgleichslosen Zuteilung von Überhangmandaten verbundene Differenzierung des Erfolgswertes der Wählerstimmen [...] in begrenztem Umfang durch das besondere Anliegen einer mit der Personenwahl verbundenen Verhältniswahl gerechtfertigt werden $\aleph^{25}$ könne, allerdings der daraus resultierende Gestaltungsspielraum des Gesetzgebers »durch den Grundcharakter der Bundestagswahl als [...] Verhältniswahl begrenzt « ${ }^{26}$ werde. Schließlich widerspreche das regelmäßige Anfallen von Überhangmandaten in größerer Zahl »der Grundentscheidung des Gesetzgebers». ${ }^{27}$ Zur Frage, wann dies der Fall sei, erklärte das Bundesverfassungsgericht in seinem Urteil vom 10. April 1997, dass es »in erster Linie Sache des Gesetzgebers « sei, darüber zu entscheiden, »ob Anlass für eine Änderung des Wahlrechts « bestehe bzw. »in welchem Ausmaß eine Erhöhung der Sitzzahl nach der in $\$ 6$ Abs. 5 BWG getroffenen Regelung hinzunehmen «sei, und dafür das Fünfprozentquorum »- bezogen auf die reguläre Gesamtzahl der Parlamentssitze - als Anhalt dienen «möge. ${ }^{28}$ Diese Entscheidung ist zwar nur von vier Richtern getragen, in der einstimmig ergangenen Entscheidung vom 3. Juli $2008^{29}$ aber nicht korrigiert worden. In seiner ebenfalls einstimmig ergangenen Entscheidung vom 25. Juli 2012 erklärte das Bundesverfassungsgericht allerdings, dass sich der Fall, dass Überhangmandate regelmäßig in größerer Zahl anfallen, »- entgegen der Ansicht der die Entscheidung vom 10. April 1997 tragenden Richter [...]- nicht allein in Orientierung an dem Fünf-Prozent-Quorum [...] bestimmen « lasse, ${ }^{30} »[\mathrm{~d}]$ ie verfassungsrechtliche Grenze für die ausgleichslose Zuteilung von Überhangmandaten« überschritten sei, »wenn Überhangmandate im Umfang von mehr als etwa einer halben Frak-

23 BVerfG, 2 BvF 3/11, 2 BvR 2670/11, 2 BvE 9/11 vom 25.7.2012, Rn. 108. Nach $\ 6$ Abs. $2 a$ BWG sollten die Zusatzmandate einer Partei vorrangig den Landeslisten mit Überhangmandaten (und anschließend den Landeslisten mit den höchsten Reststimmen) zugeordnet werden, wodurch Überhangmandate (mit Zweitstimmen) »unterlegt« und infolgedessen reduziert worden wären.

24 Ebd., Rn. 107.

25 Ebd., Rn. 132.

26 Ebd., Rn. 127.

27 Ebd., Rn. 141.

28 BVerfG, 2 BvF 1/95 vom 10.4.1997, Rn. 115.

29 Vgl. BVerfG, 2 BvC 1/07, 2 BvC 7/07 vom 3.7.2008.

30 BVerfG, 2 BvF 3/11, 2 BvR 2670/11, 2 BvE 9/11 vom 25.7.2012, Rn. 141. 
tionsstärke zu erwarten « seien, ${ }^{31}$ und - da die »voraussichtlich zu erwartende Zahl an Überhangmandaten [...] deutlich oberhalb der zulässigen Höchstgrenze « liege - »nunmehr eine Handlungspflicht des Gesetzgebers « bestehe. ${ }^{32}$ Diese Handlungspflicht umfasst allerdings keine komplette Vermeidung bzw. keinen vollständigen Ausgleich von Überhangmandaten. Das Bundesverfassungsgericht hat schließlich festgestellt, dass die Differenzierung des Erfolgswerts der Wählerstimmen durch ausgleichslose Überhangmandate bis zu einer halben Fraktionsstärke »durch das besondere Anliegen einer mit der Personenwahl verbundenen Verhältniswahl gerechtfertigt werden $\ll^{33}$ könne bzw. dadurch der »Grundcharakter der Bundestagswahl als Verhältniswahl «34 nicht aufgehoben werde - und zugleich auf die negativen Nebenwirkungen eines Vollausgleichs hingewiesen: »Allerdings erforderte eine vollständige Verwirklichung des Ziels der Verhältniswahl eine im Einzelnen nicht vorhersehbare Erhöhung der Sitzzahl des Bundestages, wodurch - abgesehen von damit verbundenen Praktikabilitätsproblemen - dessen Zusammensetzung das Ziel, die Abgeordneten des Deutschen Bundestages zur Hälfte personenbezogen zu legitimieren, nicht verwirklichen würde und Beeinträchtigungen des föderalen Proporzes zu erwarten wären. «35

\subsection{Ergebnis und Bewertung}

Sowohl bei der Bestimmung der Sitzkontingente der Länder ( $\$ 6$ Abs. 1 Satz 1 BWG) als auch bei der Reststimmenverwertung bzw. Vergabe von Zusatzmandaten ( $\$ 6$ Absatz 2a BWG) handelt es sich nicht um die tragenden Grundpfeiler des mit dem Neunzehnten Gesetz zur Änderung des Bundeswahlgesetzes eingeführten Sitzzuteilungsverfahrens, das in seiner Grundstruktur vom Bundesverfassungsgericht selbst - sogar wiederholt ${ }^{36}$ - neben zwei anderen Modellen als Regelungsalternative vorgeschlagen wurde. Zudem hätten sich die durch das Bundesverfassungsgericht mit Blick auf die Bestimmung der Sitzkontingente der Länder und die Reststimmenverwertung bzw. Vergabe von Zusatzmandaten beanstandeten Regelungen verhältnismäßig einfach korrigieren lassen, etwa indem man die Sitzkontingente der Länder nach der Bevölkerungszahl bestimmt und bei der Reststimmenverwertung nicht nur die Abrundungsverluste, sondern auch die Aufrundungsgewinne der Landeslisten berücksichtigt. Auf die Reststimmenverwertung bzw. Vergabe von Zusatzmandaten hätte natürlich auch gänzlich verzichtet werden können. Folglich betreffen die oben aufgeführten Beanstandungen des Bundesverfassungsgerichts eher minder bedeutende Ausgestaltungen des mit dem Neunzehnten Gesetz zur Änderung des Bundeswahlgesetzes eingeführten Sitzzuteilungsverfahrens, die entweder

31 Ebd., Rn. 127.

32 Ebd., Rn. 145.

33 Ebd., Rn. 132.

34 Ebd., Rn. 109.

35 Ebd., Rn. 135.

36 Vgl. BVerfG, 2 BvC 1/07, 2 BvC 7/07 vom 3. Juli 2008, Rn. 124; BVerfG, 2 BvC 4/04 vom 15. Januar 2009, Rn. 27.

ZfP 60. Jg. 2/2013 
verhältnismäßig einfach hätten korrigiert oder ohne größere Auswirkungen hätten weggelassen werden können.

Weitaus größere Implikationen hatte indessen die Rechtsprechung des Bundesverfassungsgerichts vom 25. Juli 2012 zur Verfassungsmäßigkeit von Überhangmandaten, bei der es überraschend von seiner früheren Rechtsprechung abgewichen ist. Die entsprechende Entscheidungsbegründung kann die Überraschung nicht schmälern, zum Teil sogar noch vergrößern - zumindest an der Stelle, wo das Gericht einräumt, dass ein von ihm vorgenommener »Akt richterlicher Normkonkretisierung nicht vollständig begründet werden kann «. ${ }^{37}$ Wenigstens wies das Bundesverfassungsgericht in seinem Urteil vom 25. Juli 2012 explizit darauf hin, dass der Gesetzgeber bislang »im Hinblick auf die vom Senat genannte Orientierung an der Fünf-Prozent-Sperrklausel [...] angesichts der Ergebnisse der Bundestagswahl im Jahre 2009 « genauso wenig zum Tätigwerden gezwungen war wie durch »die Entscheidungen des Senats zum negativen Stimmgewicht ${ }^{3} .{ }^{38}$

Bedenkt man, dass das Neunzehnte Gesetz zur Änderung des Bundeswahlgesetzes mit Blick auf die Verfassungsmäßigkeit von Überhangmandaten an einem Maßstab gemessen wurde, der zum Zeitpunkt der Entstehung und Verabschiedung des Gesetzes in dieser Form nicht existierte, und die übrigen Beanstandungen des Bundesverfassungsgerichts minder bedeutende Komponenten des reformierten Sitzzuteilungsverfahrens betrafen, die problemlos hätten korrigiert oder weggelassen werden können, kann es nur überraschen, dass die Reform vom Präsidenten des Bundesverfassungsgerichts Andreas Voßkuhle als »ernüchternd « $^{39}$ oder vom SZ-Journalisten Nico Fried als »Demonstration der Unfähigkeit « ${ }^{40}$ bezeichnet wurde.

\section{Die ernente Reform des Bundeswablgesetzes}

Grundsätzlich ist festzustellen, dass (mittlerweile) vielfältige verfassungsrechtlich notwendige und verfassungspolitisch gewollte Ziele existieren, zwischen denen zum Teil erhebliche Zielkonflikte bestehen, und es infolgedessen völlig unmöglich ist, allen (verfassungspolitisch gewollten) Zielen umfassend gerecht zu werden. Mit anderen Worten: Es gibt kein »perfektes Sitzzuteilungsverfahren« - ein Sitzzuteilungsverfahren, das die Grundarchitektur der bestehenden personalisierten Verhältniswahl bewahrt, den Effekt des negativen Stimmgewichts vollständig verhindert, Überhangmandate komplett vermeidet bzw. ausgleicht, den bundesweiten Parteienproporz umfassend garantiert, den föderalen Proporz (den Proporz zwischen den Ländern bzw. zwischen den Landeslisten einer Partei) restlos wahrt, Reststimmen von Landeslisten verwertet, die Bundestagsgröße nicht anwachsen lässt und obendrein noch leicht verständlich ist. Da ein Sitzzu-

37 BVerfG, 2 BvF 3/11, 2 BvR 2670/11, 2 BvE 9/11 vom 25.7.2012, Rn. 144.

38 Ebd., Rn. 151.

39 Zit. in Philipp Wittrock, »Urteil zum Wahlrecht: Karlsruhe blamiert Schwarz-Gelb « in: http:// www.spiegel.de/politik/deutschland/urteil-zum-wahlrecht-bundesverfassungsgericht-straftschwarz-gelb-ab-a-846249.html, 25.7.2012.

40 Nico Fried, »Schmetterschlag gegen Schwarz-Gelb« in: http://www.sueddeutsche.de/politik/ urteil-zum-wahlrecht-schmetterschlag-gegen-schwarz-gelb-1.1422112, 25.7.2012. 
teilungsverfahren alle oben genannten Ziele nicht (gleichermaßen) erreichen kann, sollte es auch nicht daran gemessen werden.

\subsection{Das Modell »Sitzkontingente plus Ausgleich»}

Mit dem Modell »Sitzkontingente plus Ausgleich« wird das Ziel verfolgt, an der personalisierten Verhältniswahl festzuhalten, den Effekt des negativen Stimmgewichts zu vermeiden, soweit ihn das Bundesverfassungsgericht beanstandet hat, sowie Überhangmandate vollständig auszugleichen. Zur verfassungsrechtlich gebotenen Vermeidung des Effekts des negativen Stimmgewichts »wird die mit dem Neunzehnten Gesetz zur Änderung des Bundeswahlgesetzes [...] eingeführte länderweise Verteilung der Sitze auf die Landeslisten der Parteien in modifizierter Form als erste Stufe der Sitzverteilung beibehalten ${ }^{41}$ Zum vollständigen Ausgleich von Überhangmandaten »wird in einer zweiten Stufe der Sitzverteilung die Gesamtzahl der Sitze so weit erhöht, bis bei anschließender bundesweiter Oberverteilung an die Parteien und Unterverteilung auf die Landeslisten alle Wahlkreismandate auf Zweitstimmenmandate der Partei angerechnet werden können ${ }^{4}{ }^{42}$

In der ersten Stufe der Sitzverteilung »wird zunächst die Gesamtzahl der Sitze [...] den Ländern nach deren Bevölkerungsanteil [...] und sodann in jedem Land die Zahl der dort nach Absatz 1 Satz $3^{43}$ verbleibenden Sitze auf der Grundlage der zu berücksichtigenden Zweitstimmen den Landeslisten zugeordnet ${ }^{44}$ ( $\$ 6$ Absatz 2 Satz 1 BWG - neu -). Die Bestimmung der Sitzkontingente der Länder nach der Bevölkerungszahl ist - wie oben dargestellt - verfassungsrechtlich zulässig und zudem sinnvoll, zumal auch die Verteilung der Wahlkreise auf die Länder nach der Bevölkerungszahl erfolgt ( $\$ 3$ Absatz 1 Satz 1 Nr. 2 BWG).

In der zweiten Stufe der Sitzverteilung wird nach $\$ 6$ Absatz 5 BWG - neu - die (nach $\$ 6$ Absatz 1 Satz 3 BWG - neu - bereinigte) Gesamtsitzzahl »erhöht, bis alle nach Berechnung der ersten Stufe auftretenden Überhangmandate auf Listenmandate der Partei anrechenbar sind ${ }^{45}$ Anschließend wird die nach $\$ 6$ Absatz 5 Satz 1 BWG - neu - ermittelte Gesamtsitzzahl zunächst »bundesweit [...] auf die nach Absatz 3 zu berücksichtigenden Parteien « ( $\$ 6$ Absatz 6 Satz 1 BWG - neu -) und anschließend in den Parteien »auf die Landeslisten verteilt«, wobei »jeder Landesliste mindestens die Zahl der in den Wahlkreisen des Landes von der Partei errungenen Sitze zugeteilt« wird ( $\$ 6$ Absatz 6 Satz 2 BWG - neu -). Schließlich werden »[v]on der für jede Landesliste ermittelten

41 BT-Drs. 17/11819, S. 1.

42 Ebd.

43 Danach werden von der Gesamtzahl der Sitze die Sitze erfolgreicher Wahlkreisbewerber abgezogen, die (1) nicht von einer Partei vorgeschlagen wurden, (2) von einer Partei vorgeschlagen wurden, die an der Sperrklausel gescheitert ist, und (3) von einer Partei vorgeschlagen wurden, für die in dem jeweiligen Land keine Landesliste zugelassen ist.

44 BT-Drs. 17/12417, S. 3.

45 BT-Drs. 17/11819, S. 5.

ZfP 60. Jg. 2/2013 
Sitzzahl [...] die Zahl der von der Partei in den Wahlkreisen des Landes errungenen Sitze ( $(5)$ abgerechnet« ( $(6$ Absatz 6 Satz 2 BWG - neu -).

\subsection{Die Auswirkungen des Modells "Sitzkontingente plus Ausgleich»}

\subsubsection{Grundarchitektur des Wahlsystems}

Das Modell »Sitzkontingente plus Ausgleich« führt dazu, dass die Grundarchitektur des bestehenden Wahlsystems bzw. der Grundcharakter »einer mit der Personenwahl verbundenen Verhältniswahl « ( $\$ 1$ Absatz 1 Satz 2 BWG) erhalten bleibt. Folglich wird wie im Gesetzentwurf ausgeführt - sichergestellt, dass »die Personenwahl von Wahlkreisbewerbern nach den Grundsätzen der Mehrheitswahl mit der Verhältniswahl von Landeslisten der Parteien kombiniert ist und durch Anrechnung der gewonnenen Direktmandate auf die Listenmandate der Grundcharakter der Verhältniswahl gewahrt wird $\ll .46$

\subsection{2 Überhangmandate}

Das Modell »Sitzkontingente plus Ausgleich« sorgt dafür, dass Überhangmandate vollständig ausgeglichen werden - und in der Folge der vom Bundesverfassungsgericht festgestellte verfassungswidrige Zustand behoben wird. Dies wird dadurch erreicht, dass in der zweiten Stufe des Sitzverteilungsverfahrens einer Partei in einem Land mindestens so viele Listensitze zugeteilt werden, wie sie in dem betreffenden Land Direktmandate gewonnen hat. Das führt dazu, dass Überhangmandate - vor dem Hintergrund der regulären Gesamtsitzzahl des Deutschen Bundestages - vollständig ausgeglichen bzw. vor dem Hintergrund der in der zweiten Stufe der Sitzverteilung ermittelten Gesamtsitzzahl des Deutschen Bundestages - komplett vermieden werden.

In der Folge kann der bisherige $₫ 48$ Absatz 1 Satz 2 BWG (»Nachrückverbot« bei Überhangmandaten) entfallen, da nach der zweiten Stufe des Sitzverteilungsverfahrens die gesamte Abgeordnetenzahl - wie es das Bundesverfassungsgericht für das Nachrücken von Ersatzleuten in seinem Beschluss vom 26. Februar 1998 gefordert hat ${ }^{47}$ - vom Zweitstimmenergebnis getragen wird und dadurch alle frei werdenden Sitze im Bundestag durch Listenbewerber nachbesetzt werden können. Das bedeutet, dass sich die Bundestagsgröße während einer Wahlperiode nicht mehr durch das bloße Ausscheiden von Wahlkreisabgeordneten einer Partei in einem Land mit Überhangmandaten dieser Partei verringern kann.

46 Ebd., S. 1.

47 Vgl. BVerfG, 2 BvC 28/96 vom 26. Februar 1998, Rn. 37. 


\subsubsection{Bundesweiter Parteienproporz}

Der vollständige Ausgleich von Überhangmandaten hat zur Folge, dass der bundesweite Parteienproporz garantiert wird. Schließlich entspricht nach der zweiten Stufe der Sitzverteilung (bei der die Parteien mit Blick auf ihre bundesweit zustehenden Mandate nicht hinter den Anteil zurückfallen können, der ihnen nach der ersten Stufe zusteht, und alle Direktmandate auf Zweitstimmenmandate einer Partei angerechnet werden können) »das Sitzverhältnis zwischen den Parteien vollständig dem bundesweiten Zweitstimmenverhältnis unter den Parteien «. ${ }^{48}$

In dieses Zweitstimmenverhältnis fließen auch Reststimmen der einzelnen Landeslisten ein, da die zweite Stufe der Sitzverteilung »in jedem Fall« ( $\$ 6$ Absatz 6 Satz 1 BWG - neu -) durchgeführt wird, d.h. unabhängig davon, ob die Gesamtsitzzahl aufgrund von Überhangmandaten erhöht werden muss oder nicht. Infolge des »automatischen Anlaufens « der zweiten Stufe ist eine zusätzliche Reststimmenverwertung nicht erforderlich, da sich - wie der Gesetzentwurf richtig ausführt - „durch die immer folgende bundesweite Verteilung [...] keine Rundungsverluste kumulieren können «. ${ }^{49}$

\subsubsection{Bundestagsgröße}

Der vollständige Ausgleich von Überhangmandaten kann zu einer nicht unerheblichen Vergrößerung des Bundestages führen. Letztlich ist ein vollständiger Ausgleich von Überhangmandaten - wenn man an der bisherigen Grundarchitektur der personalisierten Verhältniswahl festhält - nur zum Preis einer unter bestimmten Voraussetzungen nicht unerheblichen Vergrößerung des Bundestages möglich, welche natürlich Auswirkungen auf dessen Funktionalität und Kosten (Amtsausstattung, Abgeordnetenentschädigung, Versorgungsansprüche) hat. Darin kann man durchaus den Hauptnachteil des Modells »Sitzkontingente plus Ausgleich« sehen, wenngleich sich dieser in zweierlei Hinsicht relativieren lässt:

Zum einen hätte sich der Deutsche Bundestag bei den letzten fünf Bundestagswahlen (von denen jede zu Überhangmandaten geführt hat) bei Anwendung des Modells »Sitzkontingente plus Ausgleich « im Durchschnitt um 5,9 Prozent der regulären Gesamtsitzzahl vergrößert. Davon entfallen 2 Prozent auf die Überhangmandate und 3,9 Prozent auf die Ausgleichsmandate. Folglich hätten die Ausgleichsmandate bzw. das Modell »Sitzkontingente plus Ausgleich « im Durchschnitt der letzten fünf Bundestagswahlen nur zu einer Vergrößerung um knapp 4 Prozent der regulären Gesamtsitzzahl geführt was nicht wünschenswert ist, aber doch hinnehmbar erscheint. Am größten wäre der Deutsche Bundestag mit einer Gesamtsitzzahl von 671 Mandaten nach der Bundestagswahl 2009 gewesen, die bislang die meisten Überhangmandate hervorgebracht hat. Damit hätte sich der Bundestag um 12,2 Prozent der regulären Gesamtsitzzahl vergrößert. Davon entfallen 4,2 Prozent auf die Überhangmandate und 8 Prozent auf die Ausgleichs- 
mandate. Dabei wird allerdings auch deutlich, dass bei dieser Bundestagsgröße drei externe Überhangmandate der CSU - die aufgrund ihres (bundesweit betrachtet) relativ geringen Zweitstimmenanteils eine verhältnismäßig große »Sprengkraft« mit Blick auf Ausgleichsmandate besitzt - völlig problemlos ausgeglichen werden können. ${ }^{50} \mathrm{Natürlich}$ ist ein signifikantes Anwachsen der Bundestagsgröße über die Gesamtzahl von 671 Mandaten nicht auszuschließen, aber bei einer - vom Bundesverfassungsgericht geforderten - »an der politischen Wirklichkeit orientier $[t] e n \ll^{51}$ Betrachtung unwahrscheinlich.

Zum anderen verfügt die Bundesrepublik Deutschland im internationalen Vergleich über eines der kleinsten Parlamente, wenn man die Abgeordnetenzahl in Relation zur Bevölkerungszahl setzt. Im EU-Vergleich hat Deutschland - gemessen an der Bevölkerungszahl - gegenwärtig sogar das kleinste Parlament. Während beispielsweise ein Abgeordneter der französischen Nationalversammlung 113.341 Einwohner und ein Abgeordneter des britischen Unterhauses 96.907 Einwohner vertritt, repräsentiert ein deutscher Abgeordneter 132.006 Einwohner. Selbst bei einer Gesamtsitzzahl des Deutschen Bundestages von 671 Mandaten, würde ein deutscher Abgeordneter mehr Einwohner (121.973) repräsentieren als ein britischer oder französischer und hätte Deutschland gemessen an der Bevölkerungszahl - das zweitkleinste Parlament in der EU (hinter Spanien).

\subsubsection{Effekt des negativen Stimmgewichts}

Durch das Modell "Sitzkontingente plus Ausgleich « wird der Effekt des negativen Stimmgewichts, soweit ihn das Bundesverfassungsgericht beanstandet hat, vermieden und in der Folge der vom Bundesverfassungsgericht festgestellte verfassungswidrige $\mathrm{Zu}$ stand behoben. Dies wird dadurch erreicht, dass - im Gegensatz zu dem früheren Gesetzentwurf der SPD ${ }^{52}$ - eine vorläufige, nach Ländern getrennte Sitzverteilung (erste Stufe der Sitzverteilung) vorgenommen wird. Allerdings können gewisse »AusgleichsEffekte« eintreten. Wenn z.B. eine Partei mehr Erststimmen und in der Folge mehr Überhangmandate erhält, bekommen andere Parteien dafür mehr Ausgleichsmandate. Es ist jedoch nicht davon auszugehen, dass derartige Effekte nach der Rechtsprechung des Bundesverfassungsgerichts zur Verfassungswidrigkeit des novellierten Bundeswahlgesetzes führen.

Zum einen ist grundsätzlich fraglich, ob eine Variation der Erststimmen zum vom Bundeserfassungsgericht beanstandeten Effekt des negativen Stimmgewichts führen kann. In seinem Urteil vom 3. Juli 2008 hat das Bundesverfassungsgericht bei seiner Definition des Effekts des negativen Stimmgewichts (»dass ein Zuwachs an Zweitstimmen [sic] zu einem Verlust an Sitzen der Landeslisten oder ein Verlust an Zweitstimmen [sic]

50 Die Daten basieren auf Simulationsrechnungen des Bundeswahlleiters, bei denen die Sitzkontingente der Länder durch eine Verdoppelung der Anzahl der Wahlkreise bzw. Direktmandate in den jeweiligen Ländern ermittelt wurden.

51 BVerfG, 2 BvC 1/07, 2 BvC 7/07 vom 3.7.2008, Rn. 99.

52 Vgl. BT-Drs. 17/5895. 
zu einem Zuwachs an Sitzen der Landeslisten führen kann «) ${ }^{53}$ relativ klar auf die Zweitstimmen abgestellt. Im Urteil vom 25. Juli 2012 hat das Bundesverfassungsgericht auf diese Definition Bezug genommen, ${ }^{54}$ allerdings an anderer, zentraler Stell ${ }^{55}$ - wie oben dargestellt - nicht mehr von "Zweitstimmen «, sondern nur noch von "Stimmen « gesprochen. Dennoch ist fraglich, ob damit die Erststimmen in derselben Weise erfasst werden sollten wie die Zweitstimmen, da der Erststimme letztlich eine völlig andere Bedeutung bzw. ein völlig anderer Wert zukommt als der Zweitstimme. Schließlich wird wie das Bundesverfassungsgericht im selben Urteil ebenfalls deutlich macht - »im Grundsatz die Gesamtzahl der Sitze [...] so auf die Parteilisten verteilt, wie es dem Verhältnis der Summen ihrer Zweitstimmen entspricht [...], während die Erststimme grundsätzlich nur darüber entscheidet, welche Personen als Wahlkreisabgeordnete in den Bundestag einziehen ${ }^{56}{ }^{56}$

Zum anderen handelt es sich bei dem Effekt, dass andere Parteien mehr Ausgleichsmandate bekommen, wenn eine Partei mehr Erststimmen und in der Folge mehr Überhangmandate erhält, um einen Mechanismus, der zwangsläufig mit dem Ausgleich verbunden ist. Mit anderen Worten: Er ist geradezu das Ziel des Ausgleichs - und damit kein »willkürlicher oder widersinniger « Effekt, von dem das Sitzzuteilungsverfahren nach der Rechtsprechung des Bundesverfassungsgerichts grundsätzlich frei zu sein hat. ${ }^{57}$ Sollte das Bundesverfassungsgericht diesen Effekt verfassungsrechtlich beanstanden, würde es sich letztlich selbst widersprechen. Schließlich hätte es dann den Ausgleich von Überhangmandaten nicht für verfassungsrechtlich zulässig erklären dürfen. Genau dies hat es aber in seinem Urteil vom 25. Juli 2012 getan, in dem es darauf hingewiesen hat, dass der durch Überhangmandate »gestörte Proporz etwa durch Zuteilung von Ausgleichsmandaten wiederhergestellt werden könnte.$^{58}$ Vor dem Hintergrund der Tatsache, dass laut Bundesverfassungsgericht die Zahl ausgleichsloser Überhangmandate den Umfang von mehr als etwa einer halben Fraktionsstärke nicht überschreiten dürfe, der Gesetzgeber aufgrund der voraussichtlich zu erwartenden Zahl an Überhangmandaten handeln müsse und dabei ein Ausgleichsmodell einführen könne, ist der im Gesetzentwurf vertretenen Ansicht zu folgen, dass »[e]ine Interpretation der Definition des negativen Stimmgewichts, nach der die Zuteilung von Ausgleichsmandaten als solche verfassungswidrig wäre, $[\ldots]$ nicht zugrunde gelegt werden ${ }^{59}$ könne.

\subsubsection{Föderaler Proporz}

Das Modell »Sitzkontingente plus Ausgleich « führt dazu, dass gravierende Verzerrungen des föderalen Proporzes vermieden werden. Schließlich wird der föderale Proporz besser

53 BVerfG, 2 BvC 1/07, 2 BvC 7/07 vom 3.7.2008, Ls, Tenor Nr. 1, Rn. 76, 130, 140.

54 Vgl. BVerfG, 2 BvF 3/11, 2 BvR 2670/11, 2 BvE 9/11 vom 25.7.2012, Rn. 16.

55 Vgl. ebd., Rn. 85.

56 Ebd., Rn. 112.

57 Vgl. ebd., Rn. 57.

58 Ebd., Rn. 135.

59 BT-Drs. 17/11819, S. 6.

ZfP 60. Jg. 2/2013 
gewahrt als bei Sitzzuteilungsverfahren, die eine Kompensation von Überhangmandaten vorsehen..$^{60}$ Natürlich wird »nicht auch innerhalb der Parteien der Vorteil derjenigen Landeslisten ausgeglichen, bei denen Wahlbewerber der Partei mehr Direktmandate gewonnen haben als nach der ersten Stufe Listenmandate auf die Landesliste entfallen wären ${ }^{61}$ Schließlich würde ein Ausgleich der durch Überhangmandate entstandenen Verzerrung des föderalen Proporzes den Bundestag auf eine Größe anwachsen lassen, die weder vertretbar noch funktionsfähig wäre. Dabei ist zu bedenken, dass auch nach dem »alten Sitzzuteilungsverfahren «, d.h. nach dem Sitzzuteilungsverfahren vor dem Neunzehnten Gesetz zur Änderung des Bundeswahlgesetzes, Überhangmandate den föderalen Proporz beeinträchtigt haben - im Gegensatz zu Kompensationsmodellen allerdings nicht doppelt, sondern nur einfach, gewissermaßen als »Draufgabe « zum proportional zustehenden Anteil, der jeder Partei in jedem Land garantiert zugeteilt wurde. Das bedeutet, dass keine Landesliste einer Partei hinter den Sitzanteil zurückfallen konnte, der ihr nach Zweitstimmen zustand. Dies ist im Modell "Sitzkontingente plus Ausgleich « ebenso, wenn man die reguläre Gesamtsitzzahl des Deutschen Bundestages zugrunde legt und von dem Verteilungsmechanismus der zweiten Stufe der Sitzverteilung ausgeht (also Überhang- und Ausgleichsmandate als »Draufgabe« betrachtet). Von dem Verteilungsmechanismus der ersten Stufe darf deshalb nicht ausgegangen werden, da die zweite Stufe die maßgebliche(re) Stufe der Sitzverteilung bildet und - aufgrund der unterschiedlichen Verteilungsmechanismen - die Landesliste einer Partei in der zweiten Stufe der Sitzverteilung hinter den Sitzanteil zurückfallen kann, der ihr nach der ersten Stufe zusteht. So stünden z.B. bei der Bundestagswahl 2009 der CDU in Sachsen-Anhalt nach der ersten Stufe der Sitzverteilung sechs und nach der zweiten Stufe der Sitzverteilung fünf Mandate zu - was aber nicht auf eine Verrechnung bzw. Kompensation oder Vergabe eines »negativen Ausgleichsmandats ${ }^{62}$ sondern auf eine erneute Sitzvergabe mit einem anderen Verteilungsmechanismus zurückzuführen ist.

In diesem Zusammenhang ist darauf hinzuweisen, dass es - aufgrund der unterschiedlichen Mechanismen der ersten und zweiten Stufe der Sitzverteilung - auch ohne Überhangmandate zu (geringfügigen) Abweichungen zwischen den beiden Stufen hinsichtlich der Sitzverteilung kommen kann. Zum einen können die unterschiedlichen Mechanismen der ersten und der zweiten Stufe der Sitzverteilung auch ohne Überhangmandate grundsätzlich zu unterschiedlichen bundesweiten Sitzanteilen der Parteien führen. So könnten einer Partei nach der zweiten Stufe weniger Mandate zustehen als nach der ersten Stufe der Sitzverteilung - wodurch es auch ohne Überhangmandate zu einer Erhöhung der Gesamtsitzzahl kommen müsste. Zum anderen können die unterschiedlichen Verteilungsmechanismen auch ohne Überhangmandate bzw. Erhöhung der Gesamtsitzzahl zu (geringfügig) unterschiedlichen Sitzzahlen für die Länder (Ländersitzkontingente / Ländersitzzahlen) bzw. für die Landeslisten einer Partei führen. ${ }^{63}$

60 Vgl. z.B. BT-Drsn. 17/5896; 17/4694; 17/11821.

61 BT-Drs. 17/11819, S. 6.

62 Vgl. Friedrich Pukelsheim in: A-Drs. 17(4)634 A, S. 6.

63 Vgl. ebd., S. 19, 22. 
3.2.7 Auswirkung auf die Verständlichkeit und Normenklarheit

Ein Nachteil des Modells »Sitzkontingente plus Ausgleich «besteht sicherlich darin, dass es relativ komplex und infolgedessen nur schwer nachvollziehbar und verständlich ist. So ist z.B. der Sinn von zwei Stufen des Sitzverteilungsverfahrens, die sich hinsichtlich des Verfahrens nicht unmaßgeblich unterscheiden, für »Nicht-Wahlrechtsexperten « nur schwer nachvollziehbar. Diese hohe Komplexität bzw. geringe Verständlichkeit ist jedoch dem Umstand geschuldet, dass eine Vielzahl verfassungsrechtlich notwendiger und verfassungspolitisch gewollter Ziele, zwischen denen zum Teil beträchtliche Zielkonflikte bestehen, erreicht bzw. zum Ausgleich gebracht werden sollen.

Im Übrigen ist festzustellen, dass die Abfassung des $₫ 6$ BWG - neu - den Anforderungen hinreichender Normbestimmtheit genügt. Dass die Neufassung »ein schwieriger Text «64 ist, liegt weniger an der Textfassung, sondern mehr an dem zu regelnden Verfahren. Letztlich muss hingenommen werden, dass äußerst komplexe Verfahren nur begrenzt verständlich geregelt werden können.

\section{Der alternative Regelungsvorschlag der Fraktion Die Linke: Das Modell »Kompensation plus Ausgleich «65}

Das Zweiundzwanzigste Gesetz zur Änderung des Bundeswahlgesetzes wurde von allen Fraktionen im Deutschen Bundestag getragen - mit Ausnahme der Fraktion Die Linke, die mit einem eigenen Gesetzentwurf einen Regelungsvorschlag einbrachte, der weitgehend dem Regelungsvorschlag des Gesetzentwurfs derselben Fraktion aus dem Jahre $2011^{66}$ entsprach und sich mit Blick auf den Kern des vorgeschlagenen Sitzzuteilungsverfahrens mit früheren Gesetzentwürfen der Fraktion Bündnis 90/Die Grünen deckte: ${ }^{67}$ das Modell »Kompensation plus Ausgleich «. Damit wurde das Ziel verfolgt, den Effekt des negativen Stimmgewichts zu beseitigen, Überhangmandate komplett zu vermeiden bzw. auszugleichen sowie eine Vergrößerung des Bundestages im Regelfall zu verhindern. Dazu war »die Anrechnung von Direktmandaten auf das Zweitstimmenergebnis einer Partei auf der Bundesebene ${ }^{68}$ vorgesehen sowie, falls darüber hinaus Überhangmandate entstehen, ein »Ausgleich, der sich nach den auf der Bundesebene erzielten Zweitstimmenanteilen der Parteien richtet «. ${ }^{69}$ Eher beiläufig wurde mit dem Gesetzentwurf das Ziel der Abschaffung der Fünf-Prozent-Hürde verfolgt, zumal erst in der Ein-

64 Wolfram Ridder, »Die erneute Reform des Bundeswahlgesetzes, Verfassungskonformer »großer Wurf oder erneutes Stückwerk? « in: GWP, 62 (2013) 1, S. 47-58, hier: S. 56.

65 Vgl. hierzu bereits: Gerd Strohmeier, Die schlechteste Wahlsystemreform - mit Ausnahme aller anderen, aaO. (FN 3), S. 406-408.

66 Vgl. BT-Drsn. 17/5896.

67 Vgl. BT-Drsn. 17/4694; 16/13658; 16/11885; 13/5575.

68 BT-Drs. 17/11821, S. 1.

69 Ebd., S. 2.

ZfP 60. Jg. 2/2013 
zelbegründung darauf hingewiesen wurde, dass mit der Neuregelung die Fünf-ProzentHürde entfalle. ${ }^{70}$

Das Modell »Kompensation plus Ausgleich « hält am Grundcharakter der personalisierten Verhältniswahl fest und führt dazu, dass Überhangmandate vollständig kompensiert bzw. ausgeglichen werden - und in der Folge der vom Bundesverfassungsgericht festgestellte verfassungswidrige Zustand behoben wird. Zum einen werden interne Überhangmandate, d.h. Überhangmandate, die bei der Unterverteilung (der Verteilung der einer Listenverbindung zukommenden Sitze auf die einzelnen Landeslisten dieser Verbindung) entstehen, kompensiert, indem (faktisch) »im Rahmen der sog. Oberverteilung die von einer Partei bundesweit errungenen Direktmandate von den dieser Partei zustehenden Gesamtmandaten abgezogen ${ }^{71}$ werden. Zum anderen werden externe Überhangmandate, d.h. Überhangmandate, die bei der Oberverteilung (der Verteilung der Mandate auf die Wahlvorschläge auf Bundesebene) entstehen, sofern sie anfallen, ausgeglichen, indem die Gesamtsitzzahl des Deutschen Bundestages um so viele Sitze erhöht wird, »wie erforderlich sind[,] um unter Einbeziehung der erzielten Überhangmandate das Verhältnis nach dem Zweitstimmenanteil der Parteien auf der Bundesebene zu gewährleisten . $^{72}$

Die vollständige Kompensation interner bzw. der vollständige Ausgleich externer Überhangmandate hat zur Folge, dass der bundesweite Parteienproporz garantiert wird. Zudem werden Reststimmen der einzelnen Landeslisten berücksichtigt, da bei dem Regelungsvorschlag davon ausgegangen wird, »dass die Landeslisten einer Partei verbunden $\sin \alpha \kappa^{73}$

Durch die vollständige Kompensation interner Überhangmandate wird eine Vergrößerung des Bundestages vermieden, nicht aber durch den vollständigen Ausgleich externer Überhangmandate. Diese sind bislang nur bei der Bundestagswahl 2009 aufgetreten, bei der die CSU erstmals drei (externe) Überhangmandate erhalten hat. Vor diesem Hintergrund wird im Gesetzentwurf davon ausgegangen, dass es durch das Modell »Kompensation plus Ausgleich «»nur in Ausnahmefällen zu einer Vergrößerung des Bundestages ${ }^{74}$ kommt und diese »insbesondere die CSU betreffen ${ }^{75}$ Diese Annahme ist in zweifacher Hinsicht zu korrigieren: Zum einen sind externe Überhangmandate, wie die letzte Bundestagswahl zeigt, im gegenwärtigen Sechs-Parteien-Parlament wahrscheinlicher denn je. Zum anderen können externe Überhangmandate nicht nur bei der CSU auftreten. So hat die CDU bei der Bundestagswahl 2009 exakt so viele Direktmandate gewonnen wie ihr bundesweit Listenmandate zustanden, und damit die Schwelle zum Gewinn externer Überhangmandate denkbar knapp verfehlt. Allerdings ist hervorzuheben, dass gerade bei der CSU die Wahrscheinlichkeit des Gewinns externer Überhang-

70 Vgl. ebd., S. 5.

71 Ebd.

72 Ebd. In der Folge ergeben sich Änderungen des $₫ 48$ Absatz 1 BWG, auf die an dieser Stelle nicht weiter eingegangen werden.

73 Ebd.

74 Ebd., S. 2.

75 Ebd., S. 5. 
mandate verhältnismäßig hoch und - wie oben dargestellt - die dabei entstehende »Ausgleichswirkung « verhältnismäßig groß ist. Infolgedessen ist es durchaus wahrscheinlich, dass das Modell »Kompensation plus Ausgleich « bei künftigen Bundestagswahlen nicht nur in Ausnahmefällen zu einer nicht unerheblichen Vergrößerung des Bundestages führen würde.

Das Modell »Kompensation plus Ausgleich « führt auch dazu, dass der Effekt des negativen Stimmgewichts, soweit ihn das Bundesverfassungsgericht beanstandet hat, vermieden wird - und in der Folge der vom Bundesverfassungsgericht festgestellte verfassungswidrige Zustand behoben wird. Durch die Kompensation interner Überhangmandate kann der Effekt des inversen Erfolgswerts nicht mehr entstehen. Allerdings können durch den Ausgleich externer Überhangmandate - wie im Modell »Sitzkontingente plus Ausgleich «-gewisse »Ausgleichs-Effekte « eintreten. So ist der oben beschriebene »Ausgleichs-Effekt « (wenn eine Partei mehr Erststimmen und in der Folge mehr Überhangmandate erhält, bekommen andere Parteien dafür mehr Ausgleichsmandate) beim Modell »Kompensation plus Ausgleich « ebenso möglich. Es ist jedoch - wie oben ausgeführt nicht davon auszugehen, dass derartige Effekte nach der Rechtsprechung des Bundesverfassungsgerichts zur Verfassungswidrigkeit des Sitzzuteilungsverfahrens führen würden.

Der Hauptnachteil des Modells »Kompensation plus Ausgleich « besteht darin, dass es den föderalen Proporz massiv verzerrt. Schließlich werden dadurch Überhangmandate, die eine Partei in einem Land erhält, mit Landeslistensitzen, die dieser Partei in einem anderen Land zustehen, kompensiert - wodurch die Verzerrung des Länder-Proporzes bzw. des parteiinternen Proporzes, die durch Überhangmandate grundsätzlich auftritt, forciert wird. So würden Landeslisten mit relativ wenigen $Z$ weitstimmen und vielen Direktmandaten »belohnt « und Landeslisten mit verhältnismäßig vielen Zweitstimmen und wenigen Direktmandaten »bestraft « werden. Im schlimmsten Fall kann dies dazu führen, dass eine Landesliste, die kein einziges Direktmandat gewonnen hat, der aber nach dem Zweitstimmenanteil Sitze zustehen, welche zur Kompensation herangezogen werden, überhaupt nicht mehr im Bundestag vertreten ist. Schließlich kann eine Landesliste nach dem Modell »Kompensation plus Ausgleich « nur nicht hinter den Sitzanteil zurückfallen, den sie als Direktmandate gewonnen hat. So wäre bei der Bundestagswahl 2009, bei der die CDU bundesweit exakt so viele Direktmandate gewonnen hat wie ihr nach dem Zweitstimmenanteil Listenmandate zustanden, kein einziger Abgeordneter mehr über die Landesliste in den Bundestag eingezogen und die CDU in Bremen überhaupt nicht mehr im Bundestag vertreten gewesen. Sowohl bei den Listenbewerbern als auch bei deren Wählern dürfte es großes Unverständnis und in der Folge ein hohes Maß an Frustration und Demotivation hervorrufen, wenn die ihnen in ihrem Land nach dem Zweitstimmenanteil zustehenden Sitze zur Kompensation von Überhangmandaten in einem anderen Land herangezogen würden. Schließlich würde dies bedeuten, dass Wähler in einem Land ihre Zweitstimme für eine Landesliste abgeben und damit faktisch einen (Wahlkreis-)Abgeordneten in einem anderen Land wählen.

Mit Blick auf die vorgeschlagene Abschaffung der Sperrklausel ist festzustellen, dass diese für die Funktions-, d.h. Handlungs- und Entscheidungsfähigkeit, des Bundestages 
unabdingbar ist und - wie das Bundesverfassungsgericht in seinem Urteil vom 10. April 1997 festgestellt hat - »einen schonenden Ausgleich zwischen parteibezogener Wahlgleichheit und Funktionsfähigkeit des Parlaments «" ${ }^{76}$ herstellt. Dies ist evident sowie gemeinhin anerkannt und bedarf daher keiner ausführlicheren Erörterung. ${ }^{77}$

\section{Fazit und Ausblick}

Das Modell »Sitzkontingente plus Ausgleich « ist kein perfektes Wahlsystem - schon allein deshalb, weil es angesichts der vielfältigen verfassungsrechtlich notwendigen und verfassungspolitisch gewollten Ziele, zwischen denen zum Teil erhebliche Zielkonflikte bestehen, kein perfektes Wahlsystem geben kann. Das Modell "Sitzkontingente plus Ausgleich « ist aber eine wesentlich bessere Regelungsalternative als das Modell »Kompensation plus Ausgleich « - und zudem ein guter Kompromiss, der mit Ausnahme der Fraktion Die Linke von allen im Bundestag vertretenen Fraktionen getragen wurde. Der Hauptnachteil der Reform besteht zweifelsohne in der Möglichkeit einer nicht unerheblichen Vergrößerung des Bundestages. Es ist allerdings weder angebracht, »WorstCase-Szenarien " zu skizzieren, noch ist es angebracht, zum gegenwärtigen Zeitpunkt über eine »Reform der Reform «nachzudenken. Dennoch soll an dieser Stelle darauf hingewiesen werden, dass der zuletzt häufig gemachte Vorschlag, bei einem zu starken Anwachsen der Bundestagsgröße die Anzahl der Wahlkreise zu reduzieren, ${ }^{78}$ inakzeptabel ist: Eine Verringerung der Wahlkreiszahl führt zwangsläufig zu einer Vergrößerung der Wahlgebiete, welche wiederum zwangsläufig zu einer Verringerung der Wahlkreisbindung der Wahlkreisabgeordneten führt. Mit anderen Worten: Eine Verringerung der Wahlkreiszahl hätte negative Auswirkungen auf die politische Kommunikation zwischen dem Wahlkreisabgeordneten und seinen Wahlkreisbürgern sowie auf die Repräsentation des Wahlkreises bzw. spezifischer Wahlkreisinteressen im Parlament. Weitaus sinnvoller wäre stattdessen eine Lösung, die einen begrenzten Ausgleich von Überhangmandaten vorsieht, $\mathrm{da}$ - wie bereits dargestellt - ein vollständiger Ausgleich von Überhangmandaten weder verfassungsrechtlich notwendig noch verfassungspolitisch uneingeschränkt sinnvoll ist. Nichtsdestotrotz ist es jetzt nicht angebracht, über eine »Reform der Reform « zu diskutieren. Vielmehr ist zu wünschen, dass das deutsche Wahlrecht, zumindest was seinen Kern, das Sitzzuteilungsverfahren, anbelangt, »zur Ruhe« kommt.

\section{Zusammenfassung}

Am 25. Juli 2012 hat das Bundesverfassungsgericht erneut Teile des (2011 novellierten Bundeswahlgesetzes) für verfassungswidrig erklärt. In dem Beitrag wird zunächst das

76 BVerfG, 2 BvF 1/95 vom 10.4.1997, Rn. 115.

77 Vgl. bereits meine Stellungnahme, die ich als Sachverständiger für die öffentliche Anhörung des Innenausschusses des Deutschen Bundestages am 5. September 2011 abgegeben habe (ADrs. 17(4)327 H, S. 19).

78 Vgl. z.B. Ute Sacksofsky in: A-Drs. 17(4)634 H, S. 4. 
Urteil bzw. der daraus folgende Regelungsauftrag beschrieben. Anschließend wird das von den Fraktionen der CDU/CSU, SPD, FDP und Bündnis 90/Die Grünen eingebrachte und beschlossene Modell "Sitzkontingente plus Ausgleich« erläutert und vor dem Hintergrund der verschiedenen verfassungsrechtlich notwendigen und verfassungspolitisch gewollten Ziele bewertet. Anschließend erfolgt eine (vergleichende) Erläuterung und Bewertung des von der Fraktion Die Linke eingebrachten Modells »Kompensation plus Ausgleich «.

\section{Summary}

On the $25^{\text {th }}$ July 2012 the German constitutional court (Bundesverfassungsgericht) once again ruled parts of the (2011 revised) electoral law unconstitutional. The article illustrates the ruling of the constitutional court and its consequences. It discusses and assesses the new electoral reform initiated and implemented by the CDU/CSU, SPD, FDP and Bündnis 90/Die Grünen. In addition, it discusses and assesses the alternative model for electoral reform initiated by Die Linke in a comparative perspective.

Gerd Strohmeier, No perfect electoral system, but a good compromise - under difficult circumstances 